\title{
Correction: Epigenetic loss of AOX1 expression via EZH2 leads to metabolic deregulations and promotes bladder cancer progression
}

\author{
Venkatrao Vantaku $\cdot$ Vasanta Putluri $\cdot$ David A. Bader (1) - Suman Maity · Jing Ma $\cdot$ James M. Arnold • \\ Kimal Rajapakshe - Sri Ramya Donepudi • Friedrich-Carl von Rundstedt • Vaishnavi Devarakonda • Julien Dubrulle • \\ Balasubramanyam Karanam · Sean E. McGuire · Fabio Stossi - Abhinav K. Jain · Cristian Coarfa (1) Q Qi Cao (i) • \\ Andrew G. Sikora · Hugo Villanueva · Shyam M. Kavuri - Yair Lotan (1) - Arun Sreekumar $\cdot$ Nagireddy Putluri
}

Published online: 17 August 2020

(c) The Author(s), under exclusive licence to Springer Nature Limited 2020

Correction to: Oncogene

https://doi.org/10.1038/s41388-019-0902-7

After publication of this article, the authors noticed errors in some of the figures. In Figs. $2 \mathrm{e}, 2 \mathrm{f}-\mathrm{g}, 4 \mathrm{a}, 4 \mathrm{j}, 5 \mathrm{a}$ and $6 \mathrm{~b}$, unmatched $\beta$-actin was inadvertently used as loading control for the immunoblots. These have been corrected using repeat data from a similar set of samples and the revised Figures containing matched $\beta$-actin and their respective quantification data are included below. In Fig. 7a, the same image was inadvertently used to represent tumors 3 and 5 in the control group. This error has been corrected using original images of tumors 3 and 5 in the control group.

Additional corrections have been made in the Article and Figure legends to enhance the clarity of the description. NAD was replaced by NADP. NAD/NADP was replaced by NADP/NADPH. The description of the antibody source and dilution for the antigens PFKFB4 (Abcam, 1:1000), G6PD, and HK1 (Cell Signaling, 1:1,000) have been included in the Methods section for Western Blot. The legend for Fig. $4 \mathrm{e}$ and $4 \mathrm{j}$ has been updated. The authors have updated Supplementary Figure 6a with higher quality images of the immunoblots from replicate experiments. In addition, new Supplementary Figure 6d, containing data from replicate mouse experiments described in Figure 7, has been included to strengthen the original findings. The legend for Supplementary Figure 6 has been updated to reflect this inclusion.

The HTML and PDF versions of this article have been corrected. The scientific conclusions of this paper have not been affected. 
A)
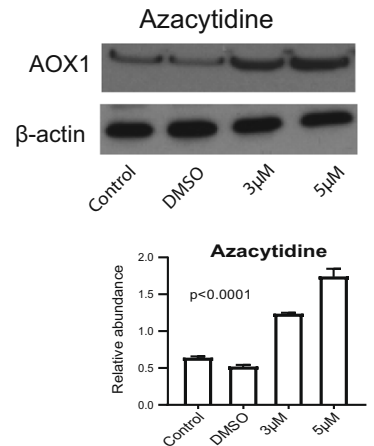

B)

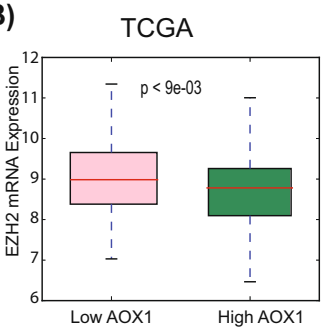

C)
Adox
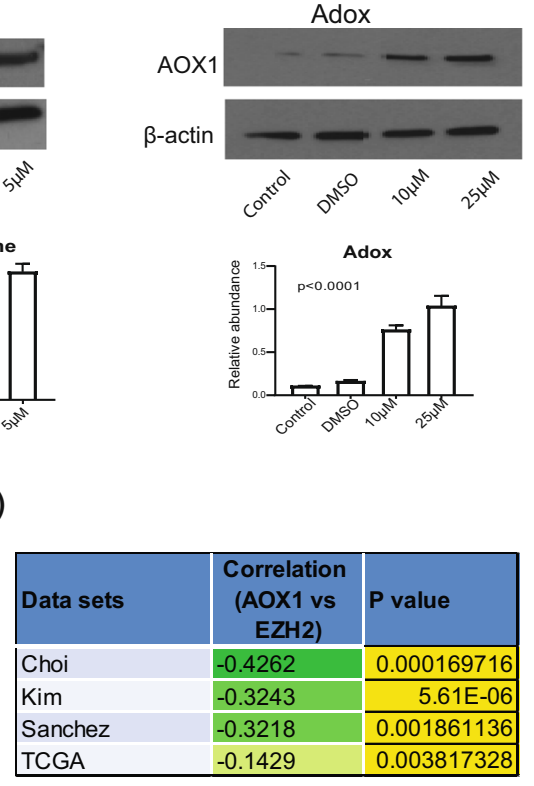

TSA
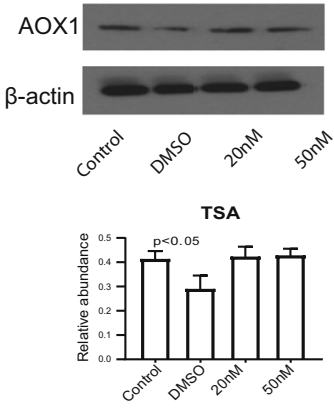

D)

AOX1

$\beta$-actin

GSK126

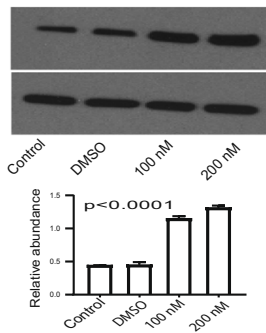

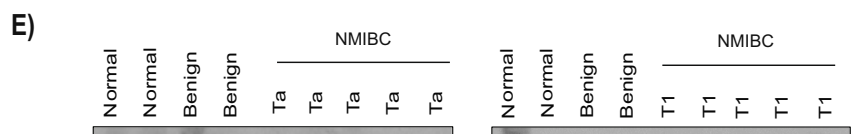

AOX1

- $0-1-$

EZH2

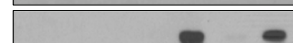

$\beta$-actin

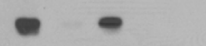

$---0-506$

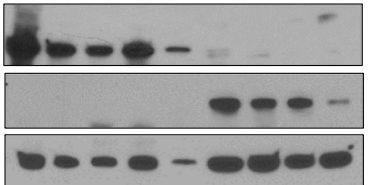

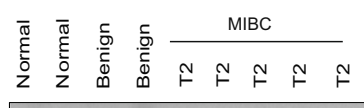
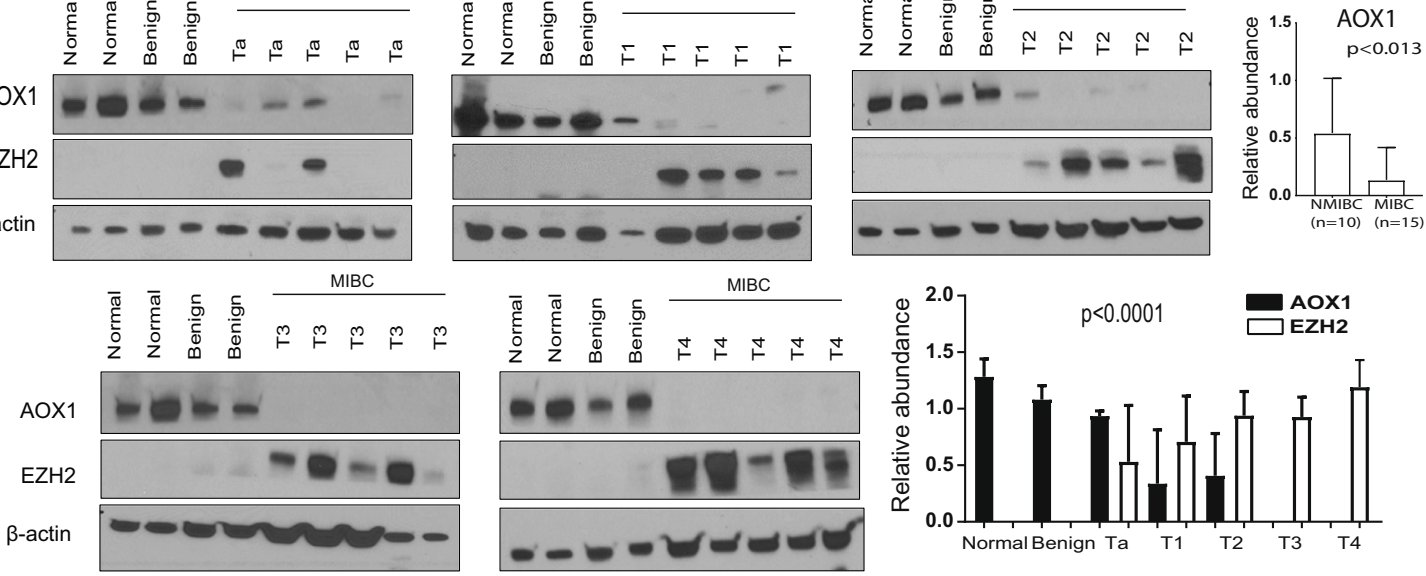

F)

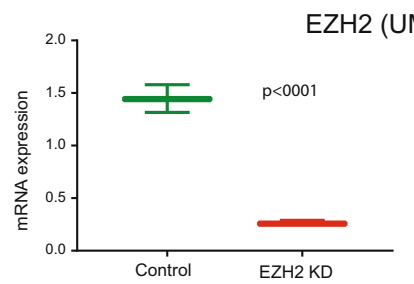

$\mathrm{EZH} 2$ (T24)

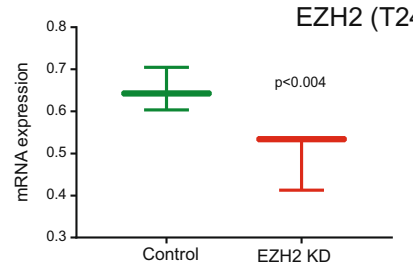

G)
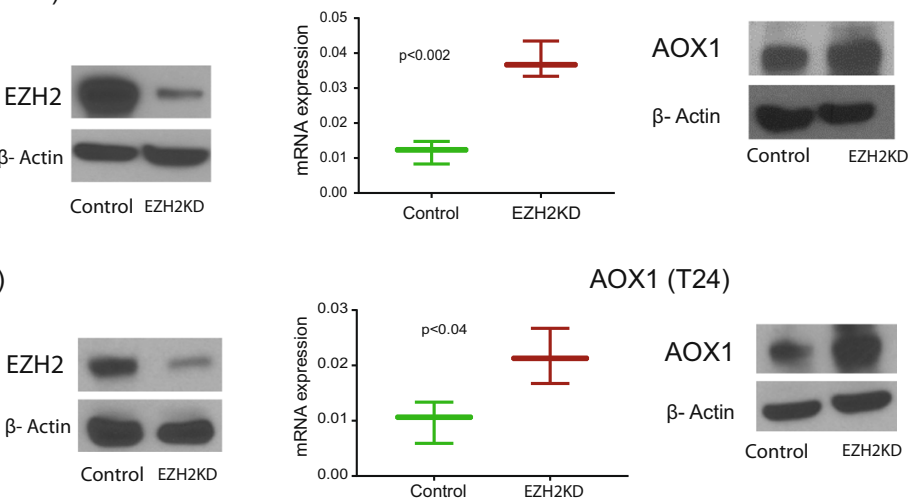

AOX1 (T24)

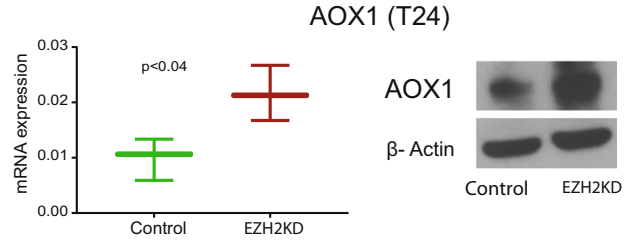

Fig. 2 
A)

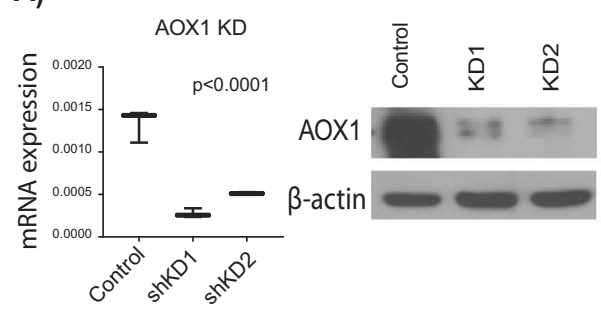

B)

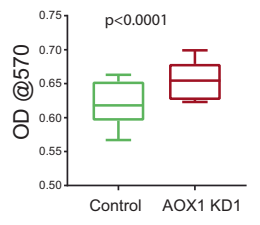

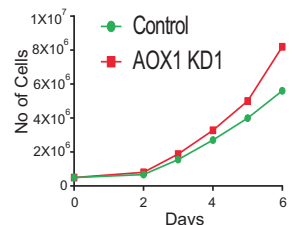

C) control

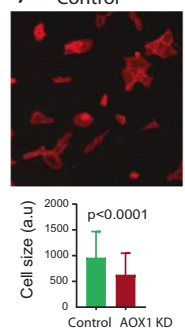

AOX1 KD1

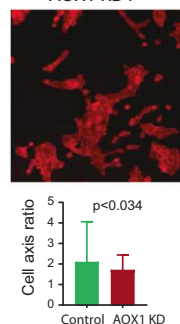

D)

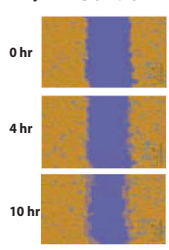

$\mathrm{AOX} 1 \mathrm{KD}$

H)

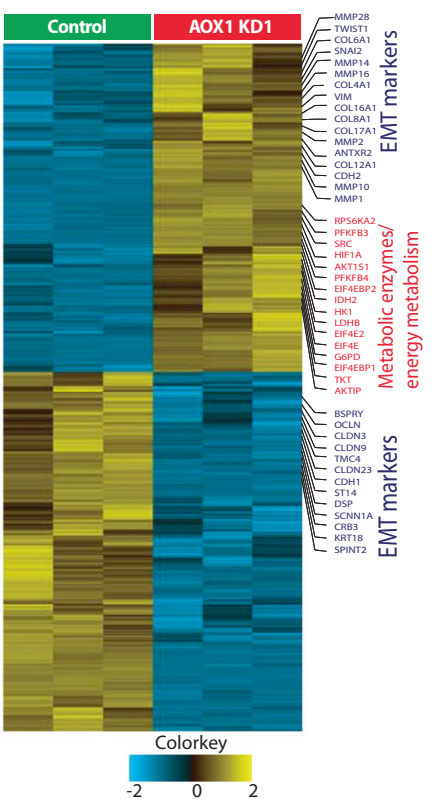

J)

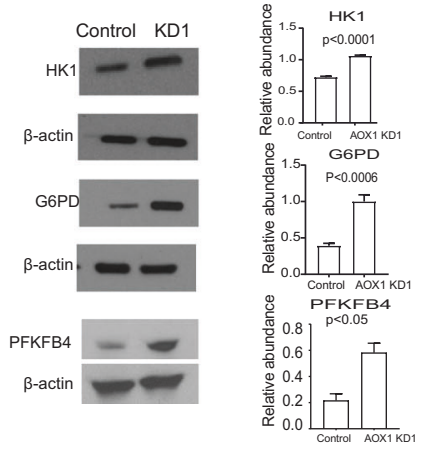

E)

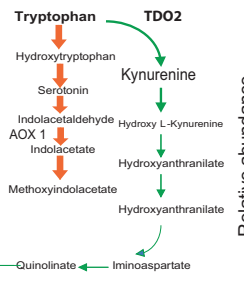

F)

F) Kynurenine NADP NADPNADPH

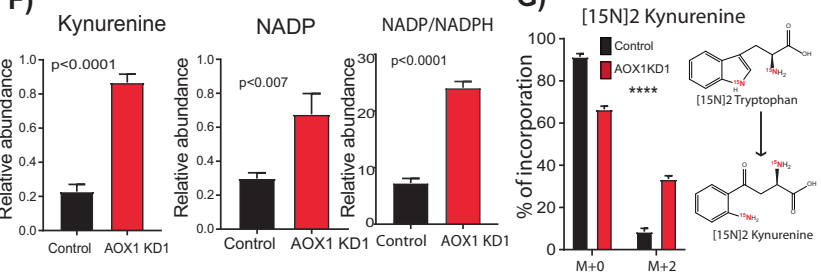

I)
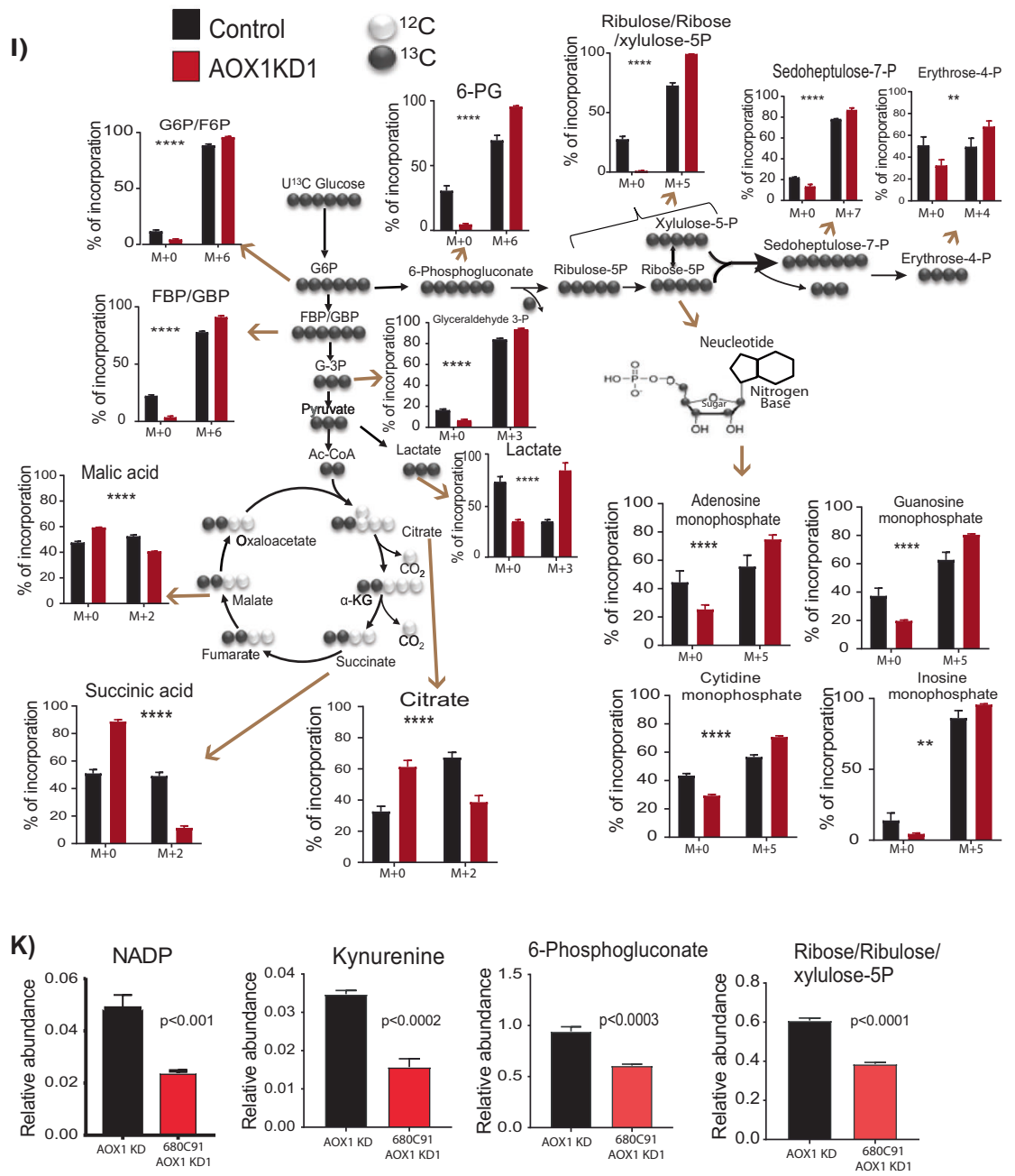

Fig. 4 


\section{A)}

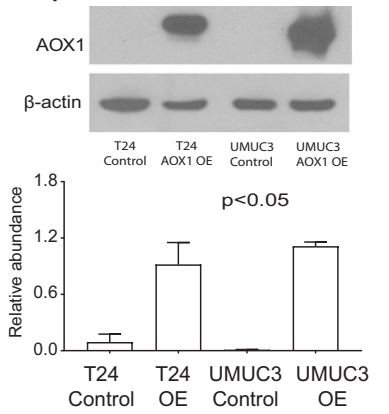

B)

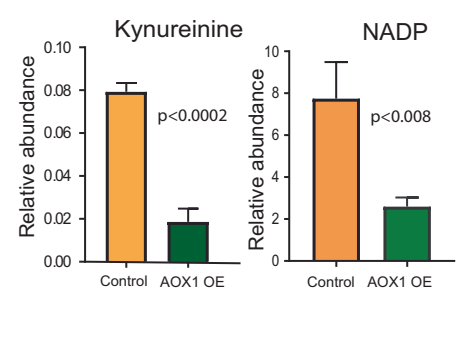

C)

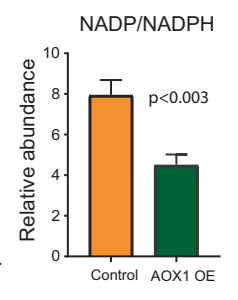

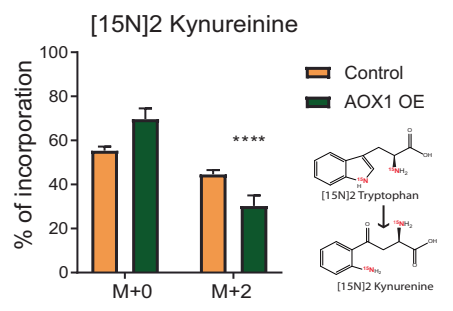

D)

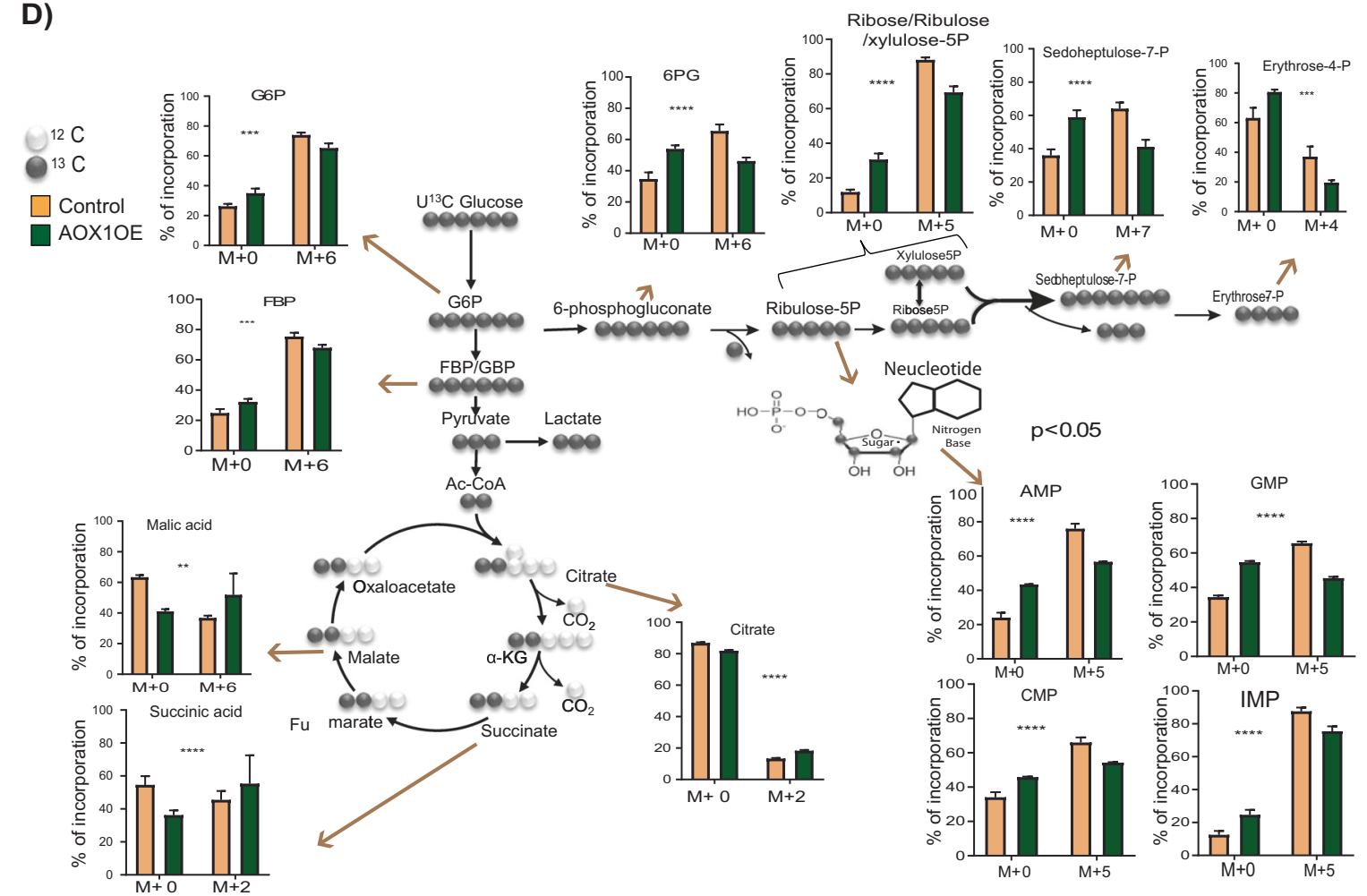

E)
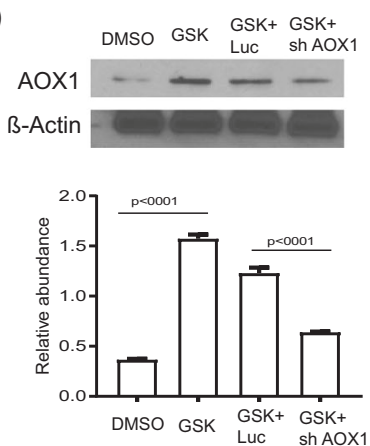

F)
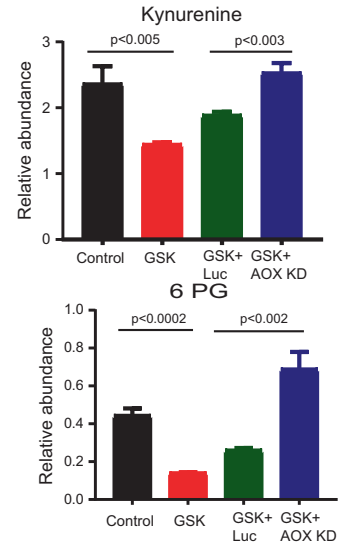
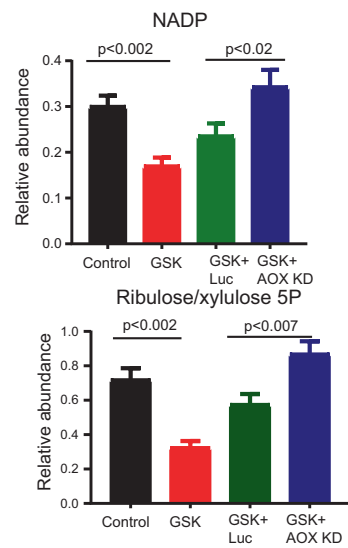

Fig. 5 
A)

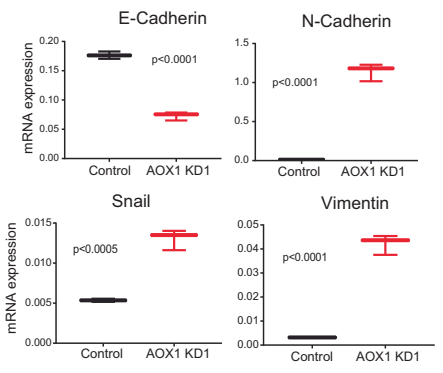

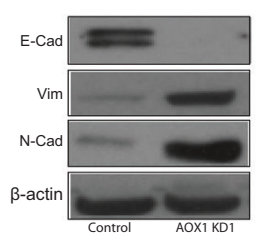

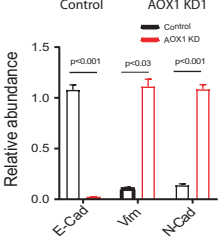

B)

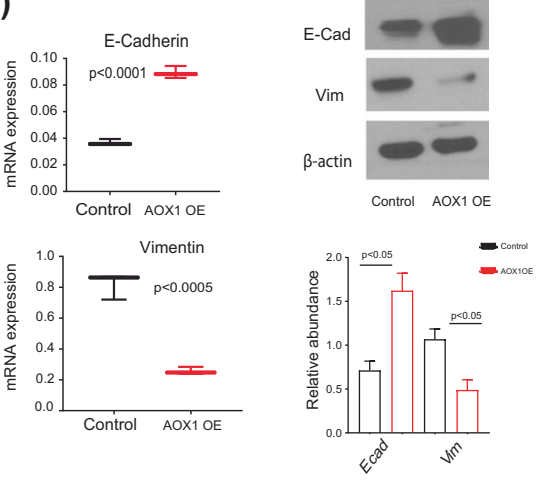

C)

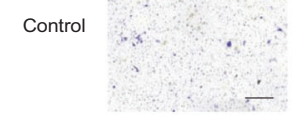

AOX1 KD1

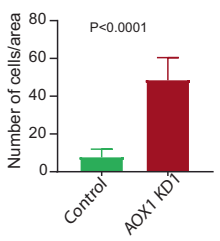

E)

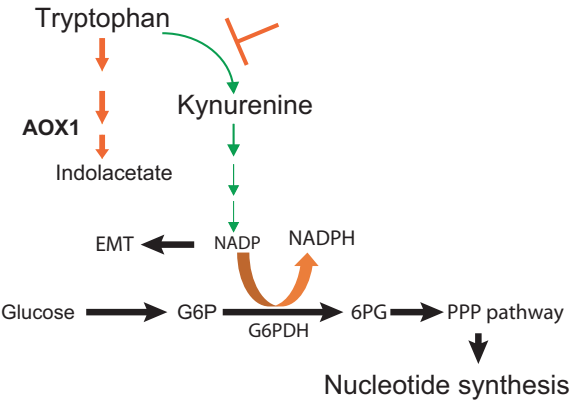

Fig. 6

D)

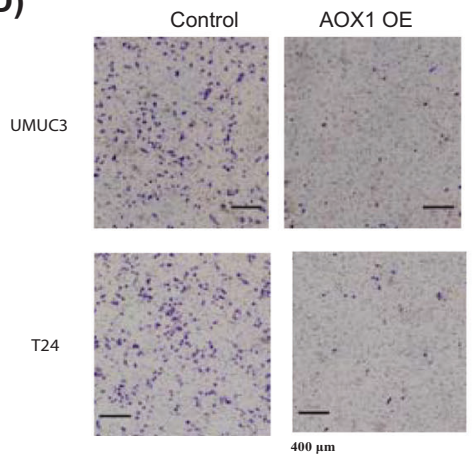

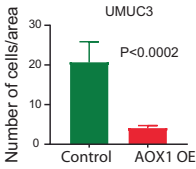

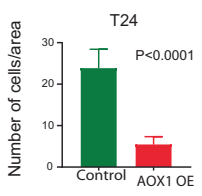

Nucleotide synthesis 
A)
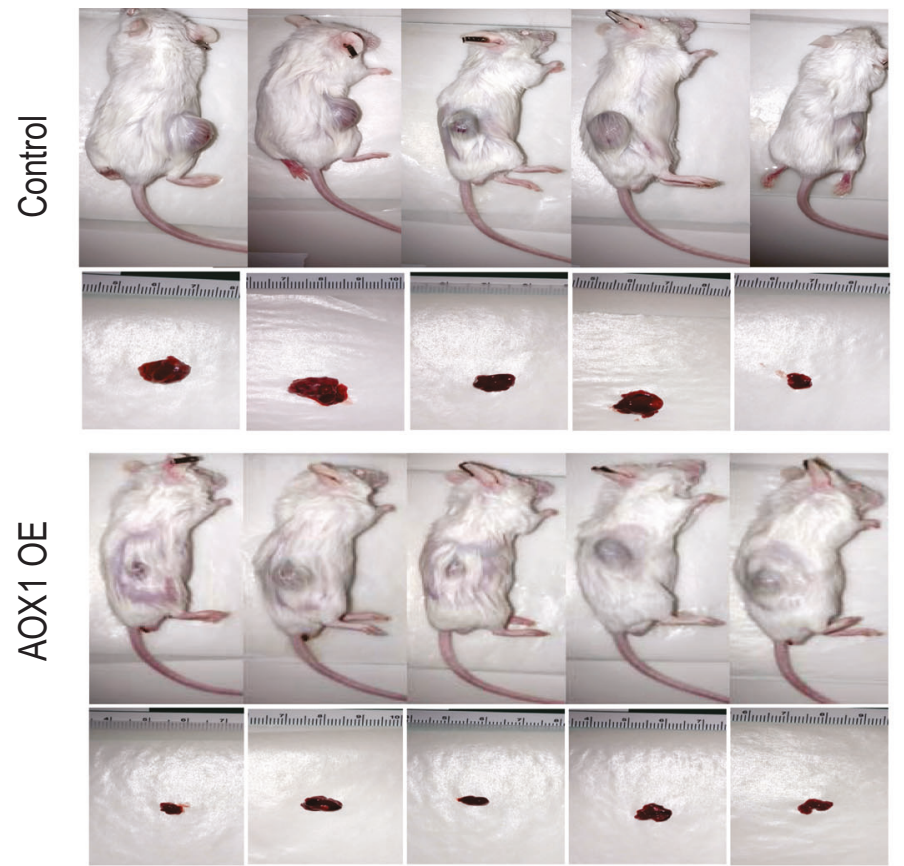

C)

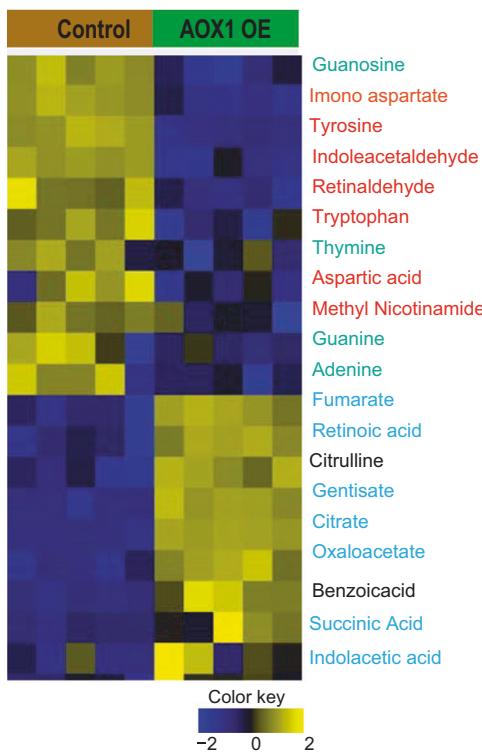

D)

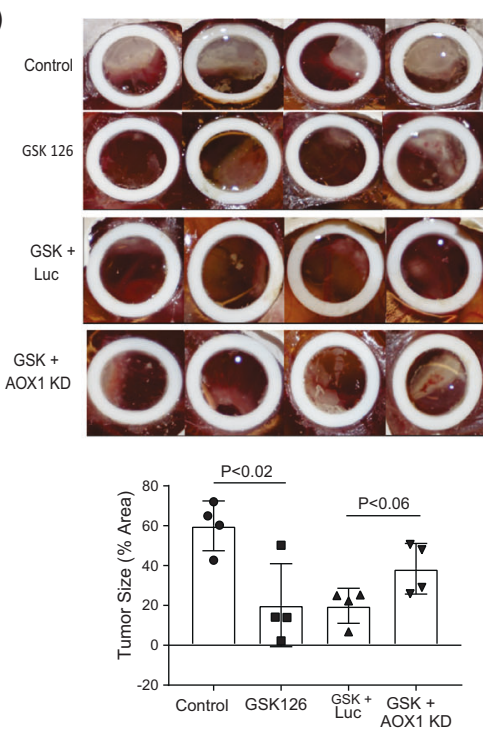

B)

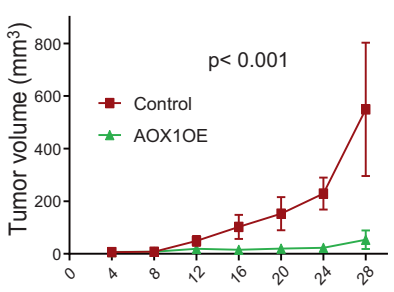

Days

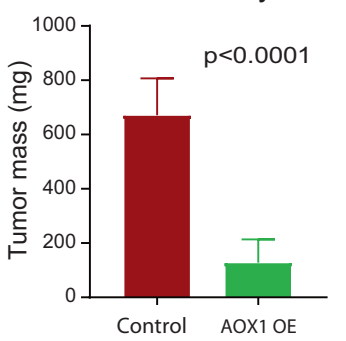

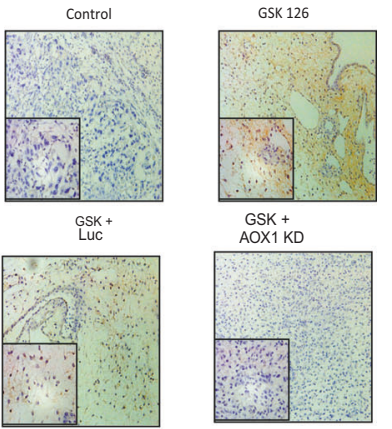

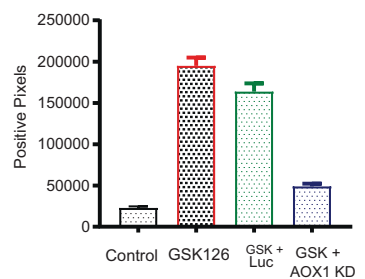

Fig. 7 